\section{Comparison of the Outcomes Between Bilateral Thoracoscopic Maximal Thymectomy Versus Trans-Sternal Maximal Thymectomy for Non- Thyomomatous Myasthenia Gravis Patients: A Local University Hospital Experience}

Waseem M. Hajjar ${ }^{1}$, Naief W. Almasry ${ }^{1}$, Abdulaziz S. Alarifi ${ }^{1}$, Fahad B. Alfahad $^{1}$, Khalid A. Alanazi $^{1}$, Abdullah F. Alfaleh ${ }^{1}$

1. College of Medicine, King Saud University, Riyadh, SAU

Corresponding author: Waseem M. Hajjar, whajjar@ksu.edu.sa

\begin{abstract}
Introduction: Myasthenia gravis (MG) is an autoimmune disease characterized by excessive and intense weakness of both respiratory and skeletal muscles. Management of MG involves both medical and surgical treatment. The surgical management includes resection of the thymus gland by many approaches, either bilateral thoracoscopic maximal thymectomy (BTT) or trans-sternal maximal thymectomy (TS). We hypothesized that bilateral thoracoscopic maximal thymectomy is as effective as trans-sternal maximal thymectomy to treat and control the disease.
\end{abstract}

Objective: This study aimed to compare the two approaches (BTT and TS) and determine which is better in terms of outcomes.

Methodology: A retrospective cohort study was conducted among 50 myasthenia gravies patients; 30 patients underwent bilateral thoracoscopic maximal thymectomy (BTT) and 20 were operated by transsternal maximal thymectomy (TS). The study was conducted at King Khalid University Hospital (KKUH), Riyadh, Saudi Arabia, between 2007 and 2017.

Result: The mean age of the MG patients was 32.6 years, ranging from 14 to 75 . Thirty-four (68\%) patients were females, and $16(32 \%)$ were males. The BTT showed less operation time $(\mathrm{P}<0.0001)$ and less intubation time (anesthesia time), which was statistically significant $(\mathrm{P}<0.0001)$. Hospital stay and ICU stay were both reported to be less in BTT (4.03 and 0.37, respectively) with p-values of 0.006 and 0.0001 , respectively.

There was no significant association between all categorical study variables and the MG patients' outcome (BTT/TS) in terms of mortality, morbidity, complete stable remission, pharmacological remission, and complications.

Review began 12/07/2020 Review ended 12/19/2020 Published 12/25/2020

\section{(๑) Copyright 2020}

Hajar et al. This is an open access article distributed under the terms of the Creative Commons Attribution License CC-BY 4.0., which permits unrestricted use, distribution, and reproduction in any medium, provided the original author and source are credited.
Conclusion: Bilateral thoracoscopic maximal thymectomy is as effective as trans-sternal maximal thymectomy to control and treat the disease.

Categories: Neurology, General Surgery

Keywords: autoimmune disease, complete stable remission, myasthenia gravis, stable pharmacological remission, surgical approaches

\section{Introduction}

Myasthenia gravis (MG) is a chronic autoimmune neuromuscular disease characterized by excessive and intense weakness in the respiratory and skeletal muscles. There are two ways to manage MG: medical and surgical [1]. Medical management includes the use of medications like pyridostigmine (cholinesterase inhibitor), corticosteroids, and azathioprine, but it does not usually show any significant improvement without other types of intervention. On the other hand, surgical management is considered the first essential and effective line in treating the thymomatous or non-thymomatous MG [2-4]. Currently, surgical management can be performed by many different surgical approaches, either by bilateral thoracoscopic maximal thymectomy (BTT) or by trans-sternal maximal thymectomy (TS) [2]. However, both approaches are effective in treating patients with MG [1-2,4]. The purpose of a maximal thymectomy operation is to remove completely the thymus gland, in addition to excise the ectopic thymic tissue, which is embedded in the mediastinal fat lying in the neck and the anterior mediastinum [5-6].

We hypothesized in this study that bilateral thoracoscopic thymectomy (BTT) for non-thymomatous MG patients is as effective as trans-sternal maximal thymectomy to treat and control the disease. We also 
hypothesized that bilateral thoracoscopic thymectomy has less complications, less morbidity and mortality, but has more cost-effective and cosmetic results.

\section{Materials And Methods}

A retrospective cohort study was carried out at King Khalid University Hospital (KKUH), Riyadh, Saudi Arabia, between 2007 and 2017 among the MG patients who underwent BTT or TS. Fifty-seven patients were evaluated; but 50 adult patients with non-thymomatous MG were included, while seven patients with thymoma or anterior mediastinal mass were excluded. All preoperative and postoperative use of medications was recorded. The comparison between the two approaches (BTT and TS) included several parameters: operation time, blood loss, hospital stay, ICU time, intubation time (anesthesia time), complications (pneumonia, wound infection etc.), diaphragmatic paresis or paralysis and vocal cord paralysis. Patients' postoperative follow up was monitored through regular visits to the outpatient's clinic post-operative for more than 24 months. Complete (cure) stable remission was also included in the comparison, which is defined as patients having no obvious MG signs or symptoms and not on any medications after six months from the surgery. Pharmacological stable remission means that the patients' signs and symptoms have been controlled after the surgery, but the patient is still on some medications. The study approval was obtained from the institutional review board (IRB), Research Ethics Committee, College of Medicine, King Saud University (KSU). The confidentiality and privacy of the patients' medical records were secured and assured.

\section{Surgical procedures}

BTT is performed while the patient is under general anesthesia, at a supine position, and intubated by a double-lumen tube with single lung ventilation. The approach is usually from the right axillary side with three ports, 5, 5, and $10 \mathrm{~mm}$. The thymus gland is dissected and excised completely, through the videoassisted thoracoscopy technique. On the left side, one $5 \mathrm{~mm}$ port is used to visualize the left phrenic nerve during dissection of the left thymic lobe.

TS is done under general anesthesia and at a supine position. A single lumen endotracheal tube is used for the patients' intubation and ventilation, a midline median sternotomy incision carried out dividing the sternum, and the two components of the thymus gland, the neck and the chest, are usually completely excised with the anterior mediastinal fat from the lower neck level down to the diaphragm. At the end of the procedure, the sternum is closed using sternal wires after draining the pleural cavity by two chest tubes.

\section{Results}

A total of 50 patients' medical records were reviewed; 34 (68\%) patients were females, and 16 (32\%) were males, with a mean age (SD) of 32.6 (12.2) years, mean body weight 74.97 (13.61) kg, and mean height 164.5 (7.69) $\mathrm{cm}$. Thirty patients (60\%) underwent BTT and 20 (40\%) underwent TS. All patients in both groups underwent a maximal thymectomy by removing completely the thymus gland in its two components, the neck and the chest with the anterior mediastinal fatty tissue. Approximately $48 \%$ of the patients were on complete stable remission, and 52\% were on stable pharmacological remission, with a mean hospital stay (SD) 5.18 (3.72) days, and a mean amount of blood loss (SD) 127.30 (74.6) ml. None of the patients from both groups had major complications, nor needed ventilator support. Two patients (4\%), one from each group, developed transient unilateral diaphragmatic paresis.

The operations took in average mean duration time (SD) 2:04 (0:78) hours, and only one patient (2\%) needed a blood transfusion. Almost all patients needed preoperative steroids (94\%), after that they were referred to ICU with a mean (SD) ICU time of 0.84 (0.933) days and intubation time mean (SD) of 2:84 (0:88) hours, and they were followed for a mean (SD) of 24.44 (20.51) months, as shown in Table 1. 


\section{Cureus}

\section{Variables}

Age (years)

32.6 (12.2)

Body weight (kg)

74.97(13.61)

Height (cm)

164.5(7.69)

Hospital stay (days)

$5.18(3.72)$

Amount of blood loss (ml)

Mean(SD)

127.30(74.6)

Operation time (hours)

2:04(0:78)

ICU stay (days)

$0.84(0.933)$

Intubation time (hours)

2:84(0:88)

Follow-up period (months)

24.44(20.51)

Gender (male)

16(32.0)

Amount of removed thymo-fatty tissue (All)

$50(100)$

Complete stable remission

24(48.0)

Pharmacological stable remission

26(52.0)

Complications (pneumonia, wound infection)

$0(0)$

Ventilator support

$0(0)$

Diaphragm paresis (transient)

2(4.0)

Vocal cord paralysis

$\mathrm{N}(\%)$

Mortality

$0(0)$

$0(0)$

Morbidity

$0(0)$

Blood transfusion

1(2.0)

Preoperative Steroid use

47(94.0)

Type of surgery ( BTT)

$30(60.0)$

Type of surgery (TS)

20(40.0)

TABLE 1: Socio demographic and clinical characteristics of myasthenia gravies patients. $(n=50)$.

(kg) kilogram, (cm) centimeter, (ml) milliliter, (ICU) intensive care unit,(sd) standard deviation, (N) number of cases, (TS) Trans-sternal maximal thymectomy, (BTT) Bilateral thoracoscopic maximal thymectomy.

The $\mathrm{t}$-test was performed to examine the difference between patients who underwent TS or BTT operations. The results were statistically significant in both operation time and intubation time, where BTT operation took much less time than TS ( $\mathrm{t}(49)=4.570, \mathrm{P}<0.0001)$, and much less intubation time $(\mathrm{t}(49)=4.690$, $\mathrm{P}<0.0001$ ). Hospital stay and ICU stay were both reported to be less in BTT 4.03, 0.37 with p-values of 0.006 and 0.0001 , respectively. The results were insignificant for all other tested variables (age, body weight, height, amount of blood loss, and follow up period), as shown in Table 2. 


\section{Cureus}

\begin{tabular}{|c|c|c|c|c|c|c|c|}
\hline \multirow{2}{*}{ Variables } & \multicolumn{2}{|c|}{ Type of surgery } & \multirow{2}{*}{ Mean difference } & \multirow{2}{*}{ t-value } & \multirow{2}{*}{ P-value } & \multicolumn{2}{|c|}{$95 \%$ Confidence interval of the mean difference } \\
\hline & TS & BTT & & & & lower & upper \\
\hline Age (years) & $32.7(13.9)$ & $32.5(11.17)$ & 0.200 & 0.056 & 0.955 & -6.955 & 7.35 \\
\hline Body weight (kg) & $74.86(12.5)$ & 75.06(14.51) & -0.202 & -0.051 & 0.960 & -8.18 & 7.78 \\
\hline Height $(\mathrm{cm})$ & 164.3(6.5) & 164.7(8.49) & -0.325 & -0.145 & 0.885 & -4.83 & 4.18 \\
\hline Operation time (hours) & $2: 56(0: 69)$ & 1:69(0:64) & 0.871 & 4.570 & 0.0001 & $0: 48$ & $1: 25$ \\
\hline Hospital stay (days) & $6.9(2.1)$ & $4.03(4.14)$ & 2.866 & 2.855 & 0.006 & 0.847 & 4.885 \\
\hline ICU stay (days) & $1.55(0.94)$ & $0.37(0.56)$ & 1.18 & 5.050 & 0.0001 & 0.703 & 1.663 \\
\hline Amount of blood loss (ml) & $140(82.97)$ & $118.8(68.7)$ & 21.166 & 0.982 & 0.331 & -22.16 & 64.49 \\
\hline Intubation time (hours) & 3:44(0:80) & $2: 45(0.69)$ & 0.991 & 4.690 & 0.0001 & $0: 566$ & $1: 41$ \\
\hline Follow up period (months) & $28.38(22.74)$ & 22.61(19.55) & 5.777 & 0.836 & 0.408 & -8.196 & 19.75 \\
\hline
\end{tabular}

\section{TABLE 2: Comparison of Mean values of quantitative study variables in relation to type of}

surgery of myasthenia gravies.

(TS) Trans-sternal maximal thymectomy, (BTT) Bilateral thoracoscopic maximal thymectomy, (kg) kilogram, (cm) centimeter, (ICU) intensive care unit, (ml) milliliter.

In both approaches, there were no statistically significant association between MG patients' outcome and all categorical study variables, such as; gender, amount of removed thymo-fatty tissue, complete stable remission, pharmacological stable remission, and most complications (pneumonia, wound infection), diaphragm paralysis or paresis, vocal cord paralysis, ventilator support, blood transfusion, preoperative steroid use, mortality, and morbidity), as shown in Table 3. 


\begin{tabular}{|c|c|c|c|c|c|c|c|}
\hline \multirow{3}{*}{ Variables } & & \multicolumn{4}{|c|}{ Type of surgery } & \multirow{3}{*}{$x^{2}$ value } & \multirow{3}{*}{ P-value } \\
\hline & & \multicolumn{2}{|l|}{ TS } & \multicolumn{2}{|c|}{ BTT } & & \\
\hline & & $\mathrm{N}$ & $\%$ & $\mathrm{~N}$ & $\%$ & & \\
\hline \multirow{2}{*}{ Gender } & Male & 6 & $37.5 \%$ & 10 & $62.5 \%$ & \multirow{2}{*}{0.061} & \multirow{2}{*}{0.804} \\
\hline & Female & 14 & $41.2 \%$ & 20 & $58.8 \%$ & & \\
\hline \multicolumn{2}{|l|}{ Complete stable remission } & 9 & $37.5 \%$ & 15 & $62.5 \%$ & 0.300 & 0.765 \\
\hline \multicolumn{2}{|l|}{ Pharmacological stable remission } & 8 & $30.8 \%$ & 18 & $69.2 \%$ & 2.739 & 0.098 \\
\hline \multicolumn{2}{|l|}{ Complications (pneumonia, wound infection) } & 0 & $0 \%$ & 0 & $0 \%$ & - & - \\
\hline \multicolumn{2}{|l|}{ Ventilator support } & 0 & $0 \%$ & 0 & $0 \%$ & - & - \\
\hline \multirow{2}{*}{ Diaphragm paresis } & Yes & 1 & $50 \%$ & 1 & $50 \%$ & \multirow{2}{*}{0.087} & \multirow{2}{*}{0.768} \\
\hline & No & 19 & $39.6 \%$ & 29 & $60.4 \%$ & & \\
\hline \multicolumn{2}{|l|}{ Vocal cord paralysis } & 0 & $0 \%$ & 0 & $0 \%$ & - & - \\
\hline \multirow{2}{*}{ Blood transfusion } & Yes & 1 & $100 \%$ & 0 & $0 \%$ & \multirow{2}{*}{1.531} & \multirow{2}{*}{0.216} \\
\hline & No & 19 & $38.8 \%$ & 30 & $61.2 \%$ & & \\
\hline \multirow{2}{*}{ Preoperative steroid use } & Yes & 18 & $38.3 \%$ & 29 & $61.7 \%$ & \multirow{2}{*}{0.946} & \multirow{2}{*}{0.331} \\
\hline & No & 2 & $66.7 \%$ & 1 & $33.3 \%$ & & \\
\hline Mortality & & 0 & $0 \%$ & 0 & $0 \%$ & - & - \\
\hline Morbidity & & 0 & $0 \%$ & 0 & $0 \%$ & - & - \\
\hline
\end{tabular}

TABLE 3: Association of categorical study variables with the type of surgery of myasthenia gravis.

(TS) Trans-sternal maximal thymectomy, (BTT) Bilateral thoracoscopic maximal thymectomy, (N) number of cases.

\section{Discussion}

Surgical management is considered one of the important keystones and essential or effective line in treating thymomatous or non-thymomatous MG patients [1].

However, there are two surgical approaches, bilateral thoracoscopic maximal thymectomy (BTT) and transsternal maximal thymectomy (TS). Few previous studies in the literature have compared both surgical approaches regarding the post-operative outcomes and the intra-operative parameters and reported different findings. Diaz and his colleagues mentioned that the video-assisted thoracoscopic surgery (VATS) approach achieved more complete stable remission than the TS maximal thymectomy approach [7]. Gung and his colleagues reported that the VATS approach has more operative time and low blood loss than the TS approach [8]. Jurado and Shiono [9,10] agreed with Gung and his colleagues [8] regarding the operative time, but on the contrary, they reported that the TS approach has more blood loss compared with the

thoracoscopic approach. Zahid et al. [3] demonstrated different results than Gung and his colleagues [8] and mentioned that VATS approach has shorter operative time compared to the TS approach. Additionally, most of the results of several studies of $[1,8,9]$ showed that VATS has less pharmacological remission and hospital stay compared to TS. Regarding the ICU stay, it was reported in most of the studies that VATS has less ICU stay [4,9]. Moreover, the incidence of complications (e.g., pneumonia) is lower in VATS and there is no significant difference in wound infection between VATS and TS [8]. Mayer et al. [2] reported that the patients who underwent TS needed more post-operative ventilation, but it was not statistically significant. Additionally, the overall mortality is higher in patients who underwent TS surgery with no statistical significance [9]. Regarding the morbidity, mortality, and post-operative complications, most of the studies found there is no difference observed between the two groups $[1,4,8,10,11]$.

The present study found that BTT has less operative time and less intubation time (anesthesia time) than TS. In addition, it has a favorable cosmetic factor. However, there was no statistical difference in complete stable remission, pharmacological remission, blood loss, and complication between the two approaches. Our study results coincide with most of the other studies, which found that the VATS group had shorter operative 
time $[3,8,10]$, but disagreed with other studies that found no time difference between the two groups $[2,6,9,11]$.

In addition, our study results showed significant statistical difference between the two approaches regarding hospital stay, which agreed with others [12-15], who reported that VATS has a shorter hospital stay. Also, the present study results show a significant statistical difference between BTT and TS in terms of ICU stay, which was found to be less in BTT and which correlated with other study results, where they found that VATS has a shorter ICU stay than TS [4,9].

The current results of our study found that there is no difference between BTT and TS concerning complete stable remission, while the results of two other studies [7,16] indicated that VATS has more complete stable remission compared to TS. Our results also reported no statistical difference between the two groups regarding pharmacological remission, which disagrees with Bachmann et al. [1], who mentioned that VATS has less pharmacological remission than TS. Additionally, in the present study, the two groups didn't show any difference regarding blood loss but the results of previous studies [12,17] reported that VATS has less blood loss compared with TS. The present study results showed no difference between the two groups regarding mortality and morbidity, which agreed with other studies $[11,18]$ that reported no difference in mortality and morbidity and agreed with several studies $[1,4,8,10]$ that found no difference between the two approaches in morbidity. Other studies $[19,20]$ reported that VATS reduced mortality.

\section{Conclusions}

BTT is as effective as TS maximal thymectomy to treat and control the disease, but it is more cost-effective than TS in terms of hospital stay, ICU stay, and also has a favorable factor on cosmetic factors. BTT has less operative time and intubation time (anesthesia time) than TS, and the results were statically significant.

There is no significant difference between the two approaches regarding complete stable remission, stable pharmacological remission, blood loss, mortality and morbidity, and all other tested variables.

\section{Additional Information \\ Disclosures}

Human subjects: Consent was obtained by all participants in this study. Animal subjects: All authors have confirmed that this study did not involve animal subjects or tissue. Conflicts of interest: In compliance with the ICMJE uniform disclosure form, all authors declare the following: Payment/services info: All authors have declared that no financial support was received from any organization for the submitted work. Financial relationships: All authors have declared that they have no financial relationships at present or within the previous three years with any organizations that might have an interest in the submitted work. Other relationships: All authors have declared that there are no other relationships or activities that could appear to have influenced the submitted work.

\section{References}

1. Bachmann K, Burkhardt D, Schreiter I, et al.: Long-term outcome and quality of life after open and thoracoscopic thymectomy for myasthenia gravis: analysis of 131 patients. Surg Endosc. 2008, 22:24702477. 10.1007/s00464-008-9794-2

2. Meyer DM, Herbert MA, Sobhani NC, et al.: Comparative clinical outcomes of thymectomy for myasthenia gravis performed by extended transsternal and minimally invasive approaches. Ann Thorac Surg. 2009, 87:385-391. 10.1016/j.athoracsur.2008.11.040

3. Zahid I, Sharif S, Routledge T, Scarci M: Video-assisted thoracoscopic surgery or transsternal thymectomy in the treatment of myasthenia gravis?. Interact Cardiovasc and Thorac Surg. 2011, 12:40-46. 10.1510/icvts.2010.251041

4. Lo C, Lu H, Hsieh M, Lee S, Chang J: Thymectomy for myasthenia gravis: video-assisted versus transsternal . J Formos Med Assoc. 2014, 113:722-726. 10.1016/j.jfma.2014.05.010

5. Ashour M: Prevalence of ectopic thymic tissue in myasthenia gravis and its clinical significance . J Thorac Cardiovasc Surg. 1995, 109:632-635. 10.1016/s0022-5223(95)70343-8

6. Siwachat S, Tantraworasin A, Lapisatepun W, Ruengorn C, Taioli E, Saeteng S: Comparative clinical outcomes after thymectomy for myasthenia gravis: thoracoscopic versus trans-sternal approach. Asian J Surg. 2018, 41:77-8510.

7. Diaz A, Black E, Dunning J: Is thymectomy in non-thymomatous myasthenia gravis of any benefit? . Interac Cardiovasc Thorac Surg. 2013, 18:381-389. 10.1093/icvts/ivt510

8. Gung Y, Zhang H, Li S, Wang Y: Sternotomy versus video-assisted thoracoscopic surgery for thymectomy of myasthenia gravis patients: a meta-analysis. Asian J Endosc Surg. 2013, 9:285-294. 10.1111/ases.12300

9. Jurado J, Javidfar J, Newmark A, et al.: Minimally invasive thymectomy and open thymectomy: outcome analysis of 263 patients. Ann Thorac Surg. 2012, 94:974-982. 10.1016/j.athoracsur.2012.04.097

10. Shiono H, Kadota Y, Hayashi A, Okumura M: Comparison of outcomes after extended thymectomy for myasthenia gravis: bilateral thoracoscopic approach versus sternotomy. Surg Laparosc Endosc Percutan Tech. 2009, 19:424-427. 10.1097/sle.0b013e3181c48242

11. Lee CY, Kim DJ, Lee JG, Park IK, Bae MK, Chung KY: Bilateral video-assisted thoracoscopic thymectomy has a surgical extent similar to that of transsternal extended thymectomy with more favorable early surgical 


\section{Cureus}

outcomes for myasthenia gravis patients. Surg Endosc. 2011, 25:849-854. 10.1007/s00464-010-1280-y

12. Fiorelli A, Mazzella A, Cascone R, et al.: Bilateral thoracoscopic extended thymectomy versus sternotomy. Asian Cardiovasc Thorac Ann. 2014, 22:1135-1137. 10.1177/0218492316647215

13. Lin MW, Chang YL, Huang PM, Lee YC: Thymectomy for non-thymomatous myasthenia gravis: a comparison of surgical methods and analysis of prognostic factors. Eur J Cardiothorac Surg. 2010, 1:7-12. 10.1016/j.ejcts.2009.05.02

14. Youssef SJ, Louie BE, Farivar AS, et al.: Comparison of open and minimally invasive thymectomies at a single institution. Am J Surg. 2010, 5:589-593. 10.1016/j.amjsurg.2010.01.001

15. Caronia FP, Fiorelli A, Arrigo E, et al.: Bilateral single-port thoracoscopic extended thymectomy for management of thymoma and myasthenia gravis: case report. J Cardiothorac Surg. 2016,11, 153. 10.1186/s13019-016-0547-3

16. Crescenzo VG, Napolitano F, Panico C, Di Crescenzo RM, Zeppa P, Vatrellad A, Laperutaa P: Surgical approach in thymectomy: our experience and review of the literature. Int J Surg Case Rep. 2017, 39:19-24. 10.1016/j.ijscr.2017.07.028

17. Hiratsuka M, Iwasaki A, Shirakusa T, Yoneda S, Yamamoto S, Shiraishi T, Tsuboi Y: Role of video-assisted thoracic surgery for the treatment of myasthenia gravis: extended thymectomy by median sternotomy vs. the thoracoscopic approach with sternal lifting. Int Surg. 2006, 1:44-51.

18. Yuan ZY, Cheng GY, Sun KL, et al.: Comparative study of video-assisted thoracic surgery vs. open thymectomy for thymoma in one single center. J Thorac Dis. 2014, 6:726-733. 10.3978/j.issn.20721439.2014.04.08

19. Xie X, Gan X, Chen B, et al.: Left- and right-sided videoassisted thoracoscopic thymectomy exhibit similar effects on myasthenia gravis. J Thorac Dis. 2016, 8:124-132. 10.3978/j.issn.2072-1439.2016.01.40

20. He Z, Zhu Q, Wen W, Chen L, Xu H, Li H: Surgical approaches for stage I and II thymoma-associated myasthenia gravis: feasibility of complete video-assisted thoracoscopic surgery (VATS) thymectomy in comparison with trans-sternal resection. J Biomed Res. 2013, 27:62-70. 10.7555/JBR.27.20120060 